\title{
Plus Shaped Chipless RFID Tag
}

\author{
Vinay Ganesh Bhagure, ${ }^{1, *}$, and Somdotta Roy Choudhury ${ }^{1}$ \\ ${ }^{1}$ R.A.I.T., Department of Electronics and Telecommunication Engineering, Nerul, Navi-Mumbai, India
}

\begin{abstract}
Chip-less radio frequency identification (RFID) technology is capable for detecting, identification, and chasing for the future Internet of Things (loT) systems and applications. The chipless RFID technology gives us the impression as a rising substitute of the traditional RFID tags and barcodes by encoding data from tags employing the radio frequency waves. The paper presents the design as well as simulation of chip-less Plus shaped RFID tags. The structure is composed of Rogers RT/Duroid 5880 by means of a substrate having the physical dimensions $40 \mathrm{~mm} \times 40 \mathrm{~mm}$ over which eight resonators are placed. This tag is compact, and the resonators are closely placed. The tag is designed using Ansys Electronics Desktop.
\end{abstract}

\section{Introduction}

Radio Frequency Identification (RFID) is a radio technology used to interconnect between two short-range objects namely, a reader and a tag. The main features of this technology are tracking, identification, and finding of objects i.e., animal's persons, logistics, etc, but more generally applications ranging from supply chain management to livestock management. RFID and its applications seem straightforward and easy. But in reality, RFID systems has foundations in many areas like system design, software development, RF and microwave theory, antenna theory, circuit theory, receiver design, integrated circuit design,encryption and network engineering, etc. The fact that RFID offers advantages over barcode systems, the cost of traditional RFID's is excessively high.

Since the presence of silicon chip has increased the prize of RFID tag, the research has been giving rise to chipless RFID technology [14][15][16]. Therefore, in chip-less RFID tags, the tag contains a printed pattern containing ID code. By using low-cost printing techniques, the prize of the tags can be therefore reduced. The aim of this paper is to present a plus shape multi resonator assembly arranged in chipless RFID tag.

\section{Design of plus shape}

A super slim double layer absorber utilizing resistively stacked frequency selective surface is introduced to give broad absorption bandwidth. Every layer has four strips of metal. The combination of metal strips and resistors on each layer are imprinted on the substrates. The structure accomplishes a broad absorption bandwidth in full-wave electromagnetic simulator.

The proposed design of structure as depicted in Fig.1, the substrate is composed of FR-4/Rogers RT Rogers $\mathrm{RT} /$ duroid 5880. On the substrate eight metal resonators are imprinted on the substrate. Relative permittivity of substrate FR-4 is 4.4, Rogers RT/duroid 5880 is 2.2 and dielectric loss tangent for both the substrate combinations is 0.02 .

Fig. 2 represents the top view of design. The geometric dimensions of substrate is: $\mathrm{L}_{\mathrm{g}}=40 \mathrm{~mm}, \mathrm{~W}_{\mathrm{g}}=40 \mathrm{~mm}$ and $\Delta=0.3 \mathrm{~mm}$. Where $\mathrm{L}_{\mathrm{g}}, \mathrm{W}_{\mathrm{g}}$ and $\Delta$ represents length, width and thickness of the substrate.

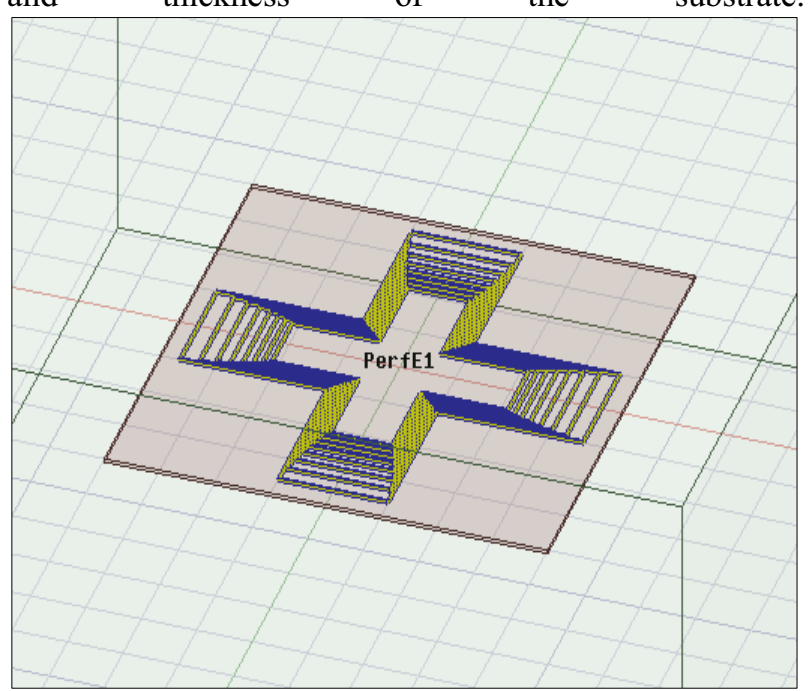

Fig. 1. 3D view of structure 


\subsection{Design Properties}

The key stages for summarizing the design of the chip-less tag are given as follows:

- Determination of the length of the $i^{\text {th }}$ resonator in vertical and horizontal directions i.e., $i=1,2,3,4 \ldots .$. , $\mathrm{n}$, according to the formulas [1]:

$$
\begin{aligned}
& L_{i}^{v}=\frac{c}{2 f_{i}^{v}} \sqrt{\frac{2}{1+\varepsilon_{r}}} \\
& L_{i}^{H}=\frac{c}{2 f_{i}^{H}} \sqrt{\frac{2}{1+\varepsilon_{r}}}
\end{aligned}
$$

Where, $\mathrm{f}_{\mathrm{i}} \mathrm{V}$ and $\mathrm{f}_{\mathrm{i}}{ }^{\mathrm{H}}$ are the $\mathrm{i}^{\text {th }}$ resonator's resonant frequency in the horizontal and vertical directions, $\varepsilon_{\mathrm{r}}$ and $\mathrm{c}$ are the relative permittivity and speed of light of the substrate.

- The design parameters are considered as per the guidelines given in the base paper which is been selected [1].

- With the help of formulas given below, length of vertical and horizontal resonators i.e., $\mathrm{L}_{\mathrm{i}}^{\mathrm{V}}$ and $\mathrm{L}_{\mathrm{i}}^{\mathrm{H}}$ can be achieved by substituting the frequency equations into length of vertical and horizontal resonators,

$$
f_{i}{ }^{H}=5 \mathrm{GHz}+(i-1) * 0.5 \mathrm{GHz}
$$

$$
f_{i}^{V}=5.05 \mathrm{GHz}+(i-1) * 0.5 \mathrm{GHz}
$$

In this tag, there is a spacing between two resonators for attaining difference of $50 \mathrm{Mhz}$ to accomplish various coding for horizontal and vertical resonators.

\begin{tabular}{|c|c|c|c|c|c|c|}
\hline Parameter & $\mathrm{Lg}$ & $\mathrm{Wg}$ & $\mathrm{L}_{\mathrm{i}}^{\mathrm{H}}$ & $\mathrm{L}_{\mathrm{i}}^{\mathrm{V}}$ & W & $\Delta$ \\
\hline Value(mm) & 40 & 40 & 18.25 & 18.07 & 0.3 & 0.3 \\
\hline
\end{tabular}

- $\quad$ The design parameters chosen for the chipless tag is,

Table 1. Selected design parameters

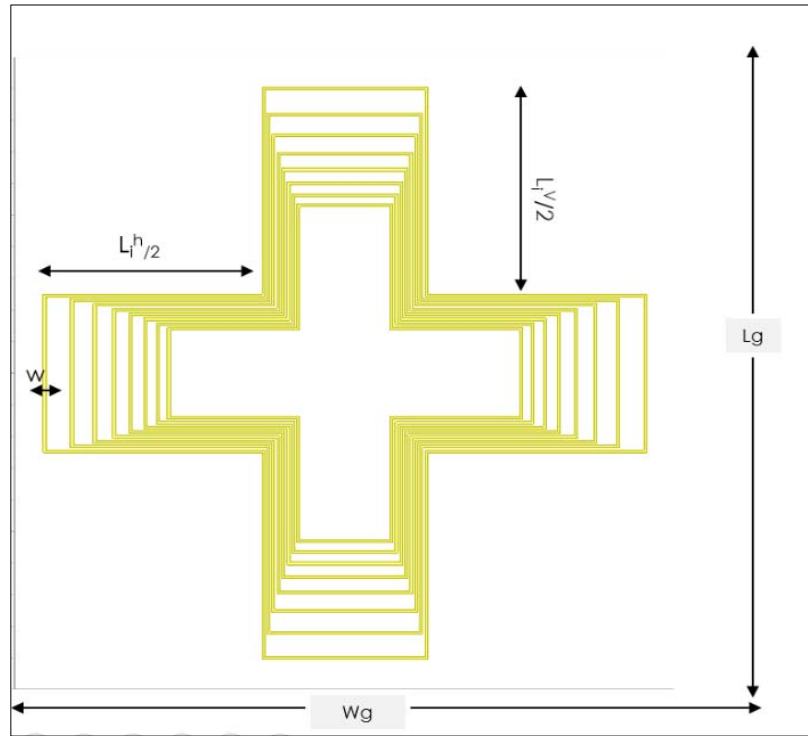

Fig. 2. Top view of design

\section{Process, simulation and analysis of chipless RFID tag}

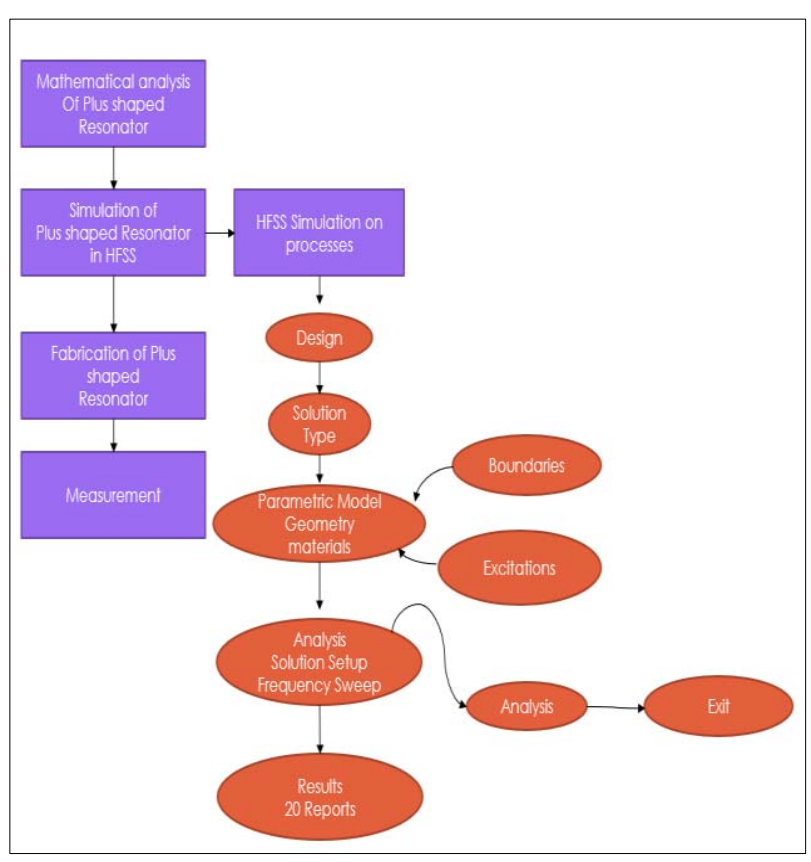

Fig. 3. Design Process

Figure 3 shows the various processes that were followed to achieve the aim of this thesis. 


\section{Simulation using two resonators}

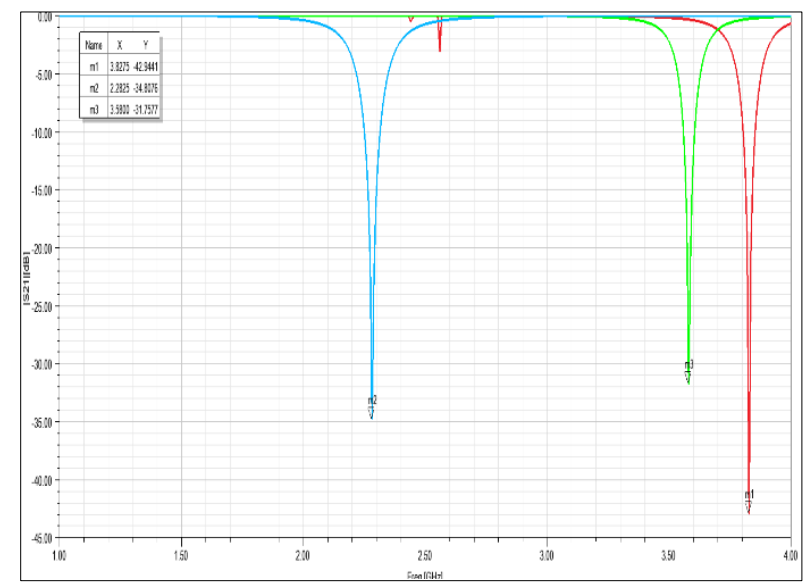

Fig. 4. Simulation result of S-parameters of 1 st and 2 nd resonator

The maximum depth observed in $|\mathrm{S} 21|$ depends on the size of the plus shape and length \& therefore in the plus shape with a length of $36.5 \mathrm{~mm}\left(\mathrm{~L}_{1}{ }^{\mathrm{H}}\right)$, maximum depth of $-34.8 \mathrm{~dB}$ is observed at resonating frequency of $2.28 \mathrm{GHz}$, while in the smaller plus size plus shape with length of $33.18 \mathrm{~mm}\left(\mathrm{~L}_{2}{ }^{\mathrm{H}}\right)$, slightly less depth of $-31.75 \mathrm{~dB}$ is observed at resonating frequency of $3.58 \mathrm{GHz}$.

When these two plus shaped resonators are kept together on the substrate depth of return loss of $-42.94 \mathrm{~dB}$ is observed in the $|\mathrm{S} 21|$, which is greater than single resonator of lengths mentioned above when placed alone on the substrate shown in figure 4.

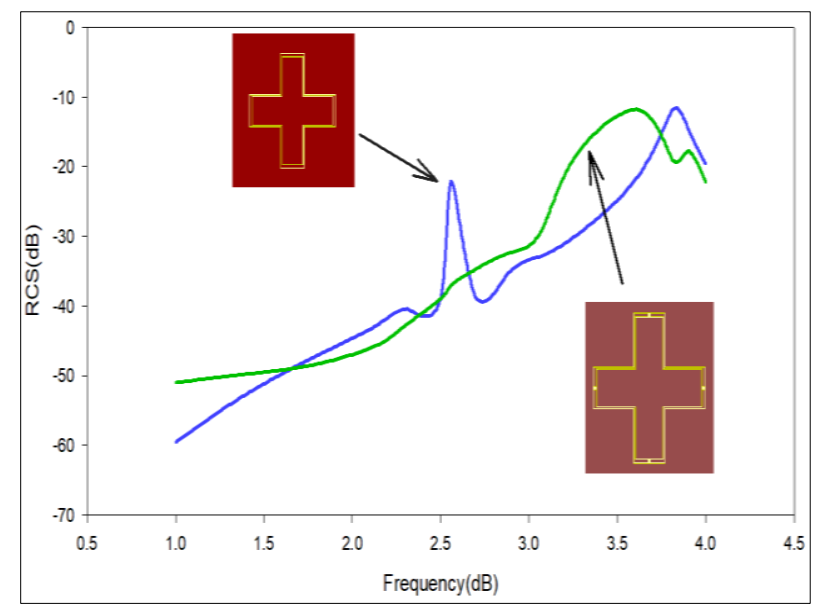

Fig. 5. RCS of 1 st and 2 nd resonator, open and short circuit

As we can see in the figure 5, due to the interaction between the adjacent rings (when the two rings are joined at all 4 sides of the plus shape), the spike is suppressed due to the contact and only single spike is observed in the monostatic RCS plot. On the other hand, when the two rings are independent (adjacent plus rings are not attached), two spikes can be seen in the RCS plot [11].

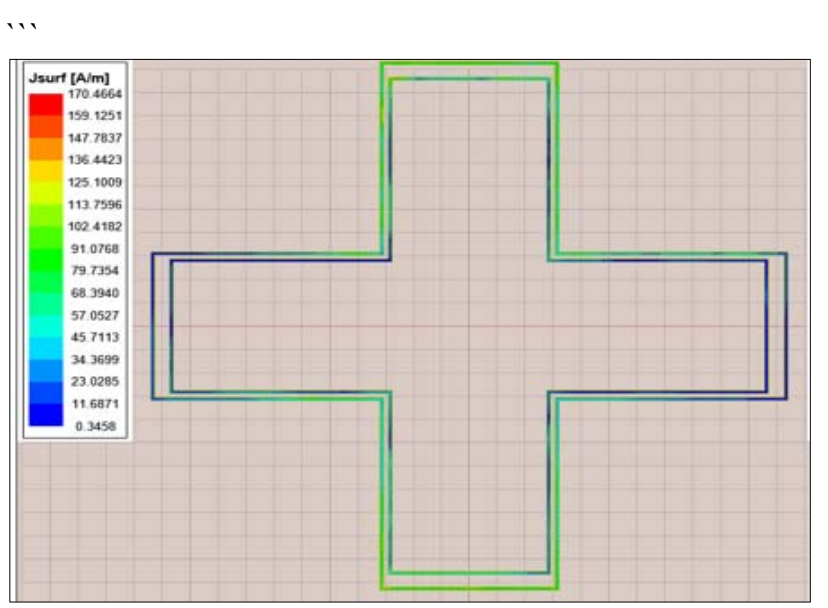

Fig. 6(a). Surface current of two resonators with open circuit

In order to input ID code to tag, adjacent rings should be attached as the above methodology is applied. First the reference tag is introduced in which all the plus shaped rings are in open circuit and we get the result as logic 1 up to 8 notches. In the second step, when we need a different coding, according to the reference tag we have to print short circuits in the assembly to suppress the resonance between two rings. Thus if, we apply short circuits between all neighbouring rings, we will get the logic 0 .

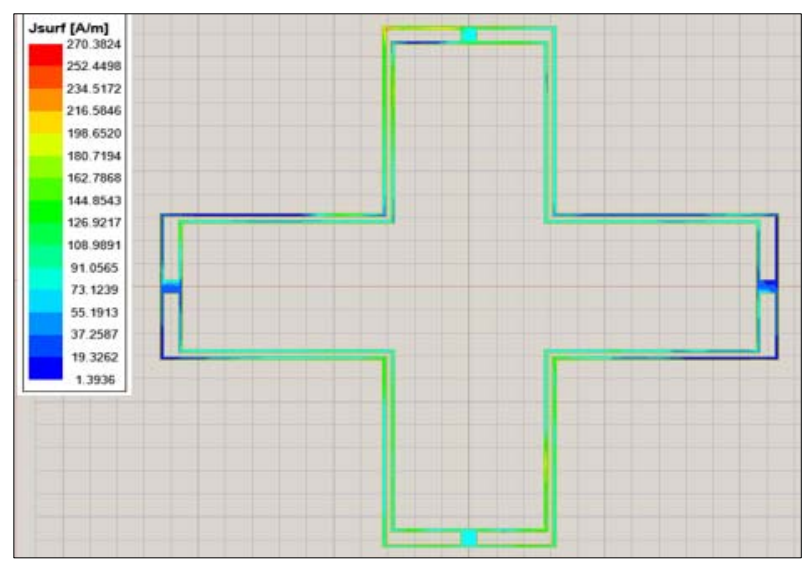

Fig. 6(b) Surface current of two resonators with closed circuit

By addition of set of short circuit's between two adjacent plus shaped, circulation of the surface currents is shown in figure 6(a) and 6(b).

These short circuits when applied on all four sides of the plus assembly. The standing waves $\left(\mathrm{J}_{\text {in }}\right.$ and $\left.\mathrm{J}_{\text {out }}\right)$ have a similar phase and therefore, the RCS produced from these sources is beneficially affected in free space, and subsequently, the resonance of the lower resonator is absent shown in figure 5 .

\section{Simulation of 8 resonators using Rogers RT/duroid 5880 substrate}


According to the figures 7(a) and 7(b) it's been seen that 8 -bits are encoded according to 8 resonances. The occurrence of single resonance will let a particular frequency to pass in result the signal being at that frequency when again transmitted to reader. The logic " 1 " can be encoded based on the resonance levels. The absence of the resonance, which can be done by removing the particular resonator from figure 3 or sorting the two resonators as mentioned above, will attenuate due to the absence of stopband. This would represent logic " 0 ". In the figure 7(b), it's been seen that the tag for the 8 resonator tag, the first 3 resonance phase jumps were expected to give return loss above $10 \mathrm{db}$. But due to less return loss, the first 3 resonators will not resonate and give Logic " 0 ".

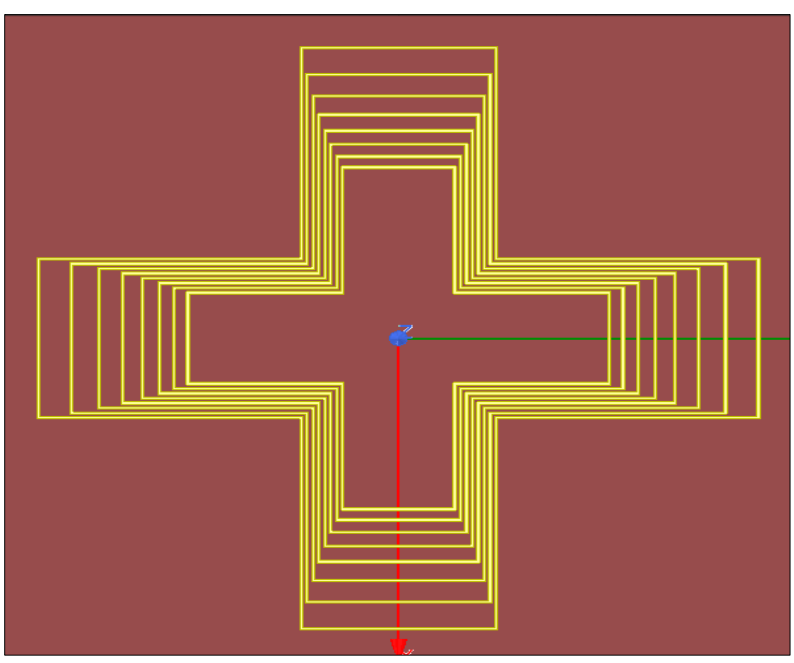

Fig. 7(a) Substrate with 8 resonators

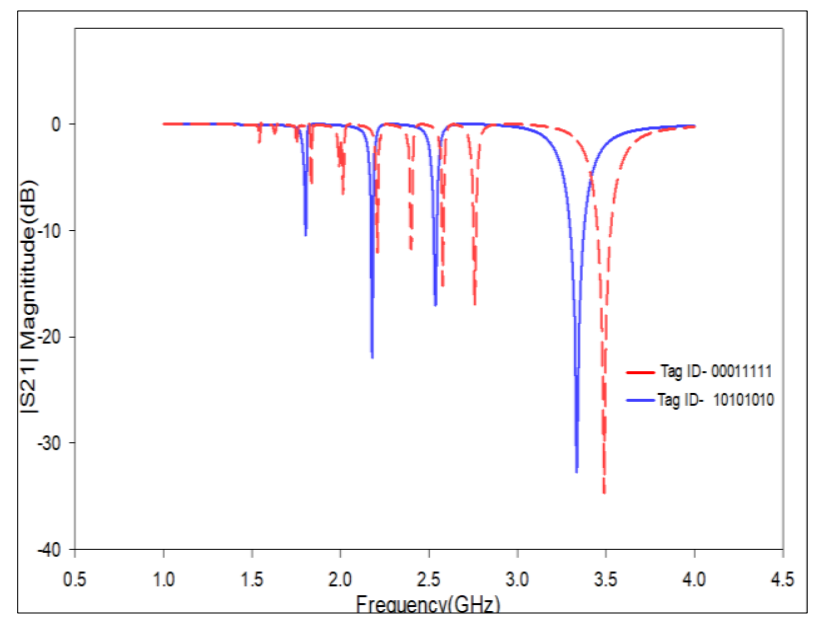

Fig. 7(b) $|\mathrm{S} 21|$ with different sets of coding

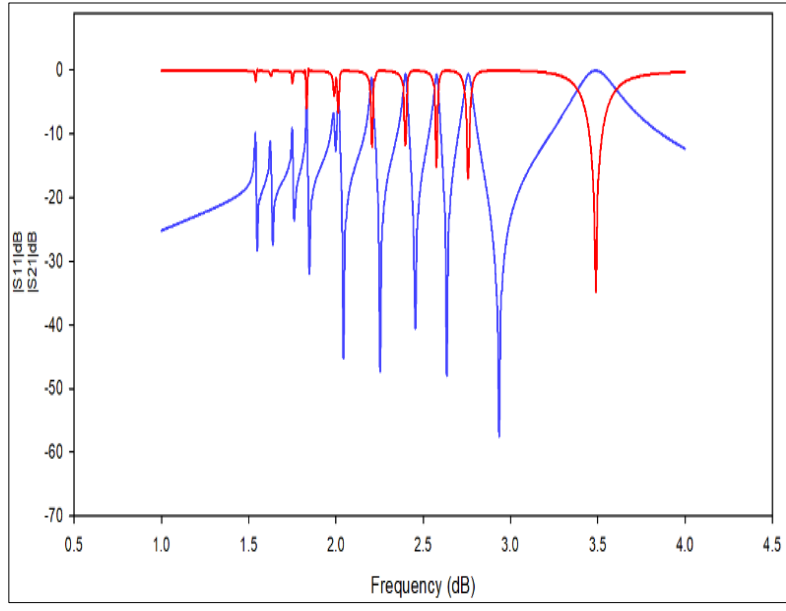

Fig. 7(c) Simulated S-parameters of 8 resonators

As mentioned above this chip-less RFID works in the frequency range of (1-4) GHz, in the figure 7(c) and figure $7(d)$ the simulated values of S parameters (S11 and S21) and the radiation pattern are shown.

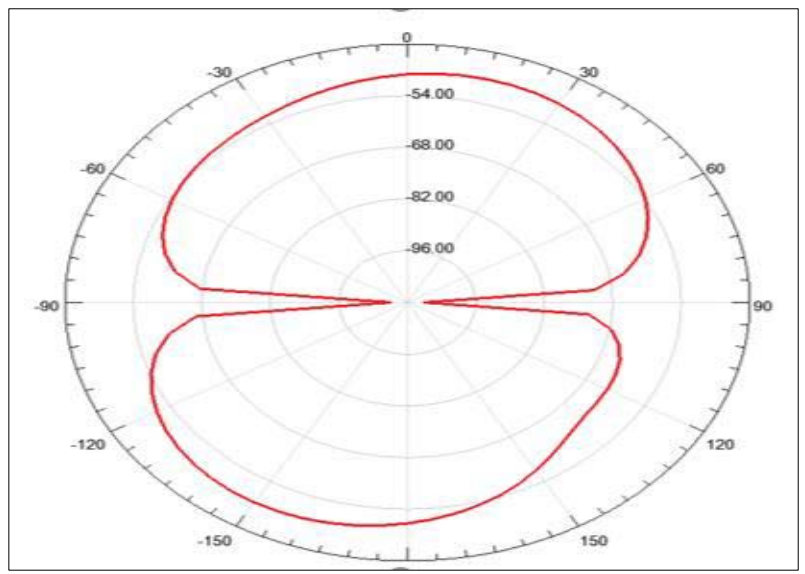

Fig. 7(d) Simulated polar plot

In order to see performance of plus shaped RFID tag, the RCS of tag is calculated at $5.5 \mathrm{GHz}$ shown in figure 8(a) and 8(b). Tags used in measurnment setup include following ID's- 11111111 and 10101010.

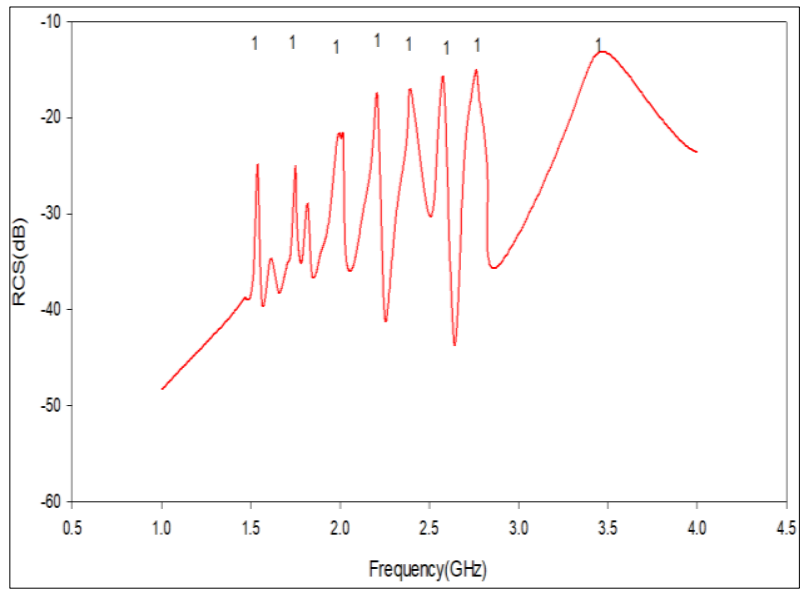

Fig. 8(a) Simulated results of RCS parameters of 8 resonators 
For all the 8 resonators combined the simulated results are shown in figure $8(\mathrm{a})$, its seen that there are 8 different resonant peaks at resonant frequency $5.5 \mathrm{GHz}$ that give us 8-bit output in RCS. On the other hand, only four resonators are taken, which all four resonators are equally spaced. Therefore, the encoded output of 4-bits is obtained which is shown in the figure 8(b) which gives is the output ID-10101010[12][13].

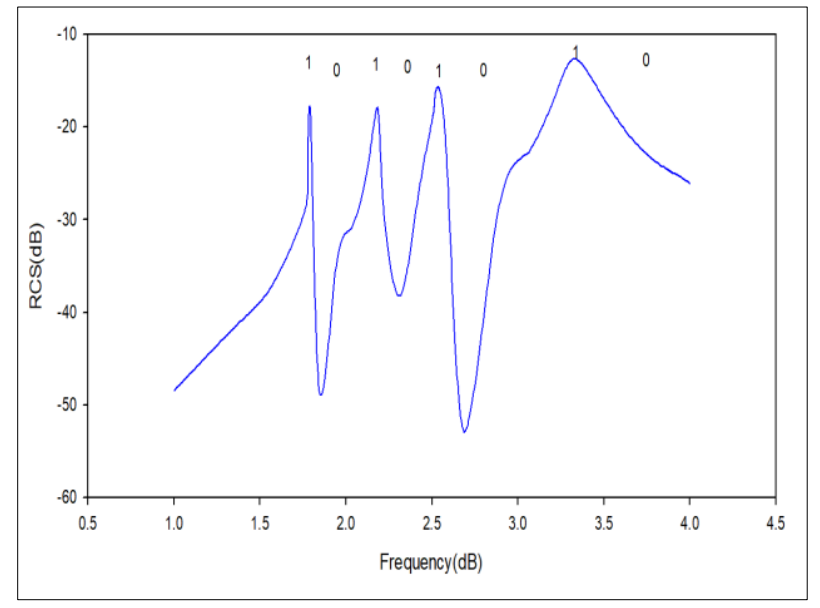

Fig. 8(b) Simulated results of RCS parameters of 4 resonators

\section{Simulation of 8 resonators using FR- 4 Substrate}

As mentioned above the chip-less tag has no ground plate included, it consists of substrate and few resonators as shown above. The study and simulation have been done by changing the substrate to FR-4. The solutions of Rogers RT-duroid 5880 substrate have been kept for reference of performance against the FR-4 substrate. The FR-4 substrate (relative permittivity $=4.4$ and dielectric loss tangent $=0.02$ ) is cheaper than Rogers RT-duroid 5880 .

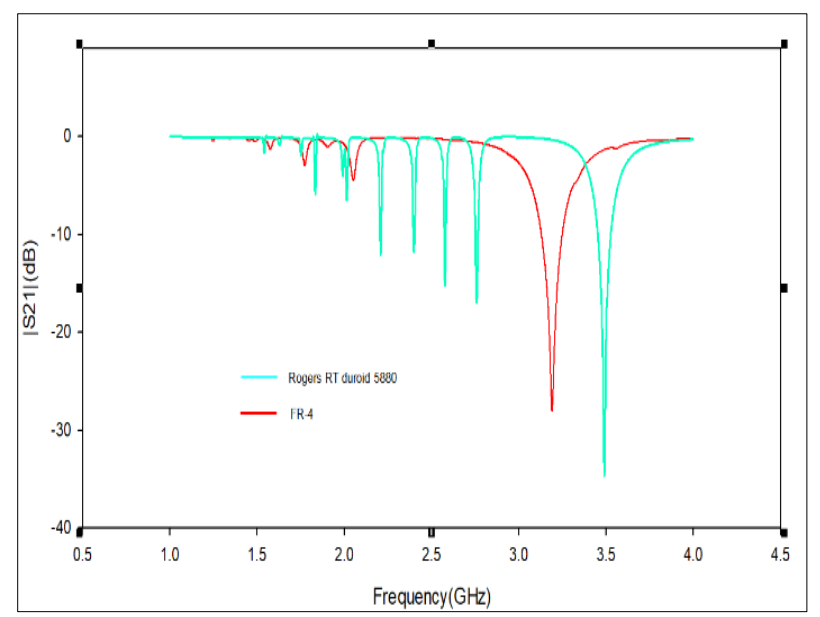

Fig. 9 Result of difference between simulation using two different substrates

As the simulation had been done with substrate as FR4 , it was observed in the return loss in the $|\mathrm{S} 21|$ graph as shown in figure 9, the return loss using the FR-4 as a substrate is very low and only 1-bit of encoded output is obtained.

On the other hand, in as compared to FR-4 substrate, 6-bit resonant peaks are obtained and the highest return loss obtained is $-37 \mathrm{~dB}$. The encoded output of the tag with Rogers RT-duroid substrate is 00111111 . The $1^{\text {st }}$ three resonators return loss is less than $-10 \mathrm{~dB}$, therefore only 6 resonators resonate at $5.5 \mathrm{GHz}$ operating frequency.

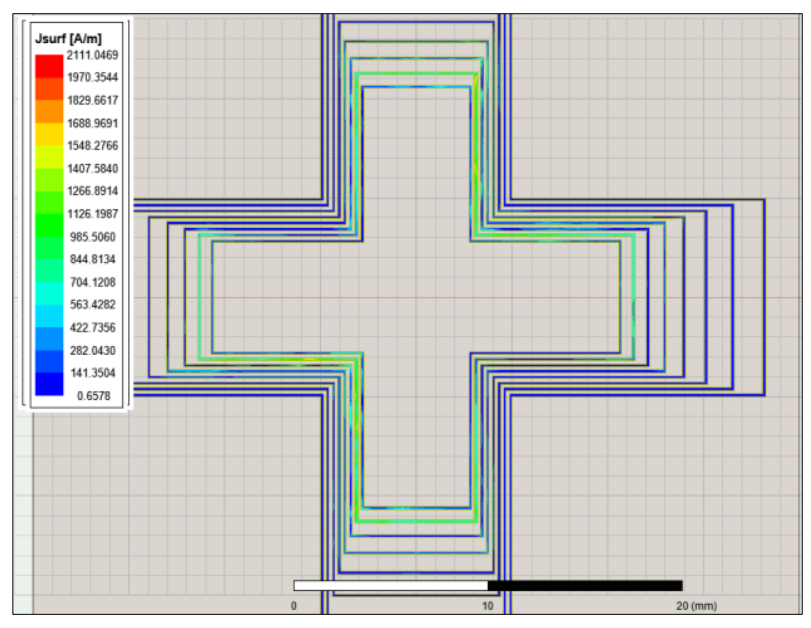

Fig. 10 Simulated result of surface current (Jsurf)

With the help of surface current distribution, the proposed structure can be explained. Here the surface current has been analyzed at the resonating frequencies 5.5 GHz. It demonstrates that the surface current is minimum in the outer resonators while the surface current is more in the inner resonators. As observed in the above figure 10 , surface current distribution is maximum in $6^{\text {th }}, 7^{\text {th }}$, and $8^{\text {th }}$ resonators where bits of green, orange and red patches can be seen on those resonators[2][3].

The Plus Shaped Chip-less tag can be fabricated using lithographic techniques [8][9][10] and inkjet printing $[4][5][6][7]$ which reduces production costs of the tag and yield high efficiency.

\section{Conclusion}

A multi-resonator structure for plus shaped chip-less RFID tags is presented. The proposed structure consists of 8 resonators. The designed chip-less RFID has uniform radiation pattern. The simulated RCS plot and measured S21 plot are similar for the proposed tag which indicates that the $\mathrm{L}$ and $\mathrm{S}$ bands of $1 \mathrm{GHz}$ to $4 \mathrm{GHz}$ can be effectively used. For generating new codes, the design of RFID tag can be modified, simply by varying the resonator's lengths by an amount equal to the slots spacing. The key performance parameters are enhanced with many simulations having resonant notches of $33 \mathrm{~dB}$ depth. The operation of the proposed structure can be explained with the help of surface current distribution in operational frequency of $5.5 \mathrm{GHz}$. The proposed tag can 
encode 6 bits. The proposed chip-less RFID tag can be incorporated in many industrial and IoT applications.

\section{References}

1. Issa, Khaled. (2017). A Novel L-Shape Ultra Wideband Chipless Radio-Frequency Identification Tag. International Journal of Antennas and Propagation.

2. P. P. Sahu, D. Prasad Mishra, T. K. Das and S. Kumar Behera, "Design of a Chipless RFID Tag for $2.4 \mathrm{GHz}$ and $5.8 \mathrm{GHz}$ ISM Band Applications," 2020 IEEE International Students' Conference on Electrical,Electronics and Computer Science (SCEECS), 2020, pp. 1-4, doi: 10.1109/SCEECS48394.2020.108.

3. O. Rance, R. Siragusa, P. Lemaître-Auger and E. Perret, "Toward RCS Magnitude Level Coding for Chipless RFID," in IEEE Transactions on Microwave Theory and Techniques, vol. 64, no. 7, pp. 2315-2325, July 2016, doi: 10.1109/TMTT.2016.2562625.

4. H. Jang, W. Lim, K. Oh, S. Moon and J. Yu, "Design of Low-Cost Chipless System Using Printable Chipless Tag With Electromagnetic Code," in IEEE Microwave and Wireless Components Letters, vol. 20, no. 11, pp. 640-642, Nov. 2010, doi: 10.1109/LMWC.2010.2073692.

5. Shao, Botao \& Chen, Qiang \& Liu, Ran \& Zheng, Li Rong. (2012). Design of fully printable and configurable chipless RFID tag on flexible substrate. Microwave and Optical Technology Letters. 54. 10.1002/mop. 26499.

6. B. Shao, Y. Amin, Q. Chen, R. Liu and L. Zheng, "Directly Printed Packaging-Paper-Based Chipless RFID Tag With Coplanar \$LC\$ Resonator," in IEEE Antennas and Wireless Propagation Letters, vol. 12, pp. 325-328, 2013, doi: 10.1109/LAWP.2013.2247556.

7. A. Vena, A. A. Babar, L. Sydänheimo, M. M. Tentzeris and L. Ukkonen, "A Novel Near-Transparent ASKReconfigurable Inkjet-Printed Chipless RFID Tag," in IEEE Antennas and Wireless Propagation Letters, vol. 12, pp. 753-756, 2013, doi: 10.1109/LAWP.2013.2270932.

8. S. Preradovic, S. M. Roy and N. C. Karmakar, "RFID System Based on Fully Printable Chipless Tag for Paper-/Plastic-ltem Tagging," in IEEE Antennas and Propagation Magazine, vol. 53, no. 5, pp. 15-32, Oct. 2011, doi: 10.1109/MAP.2011.6138421.

9. A. Vena, E. Perret and S. Tedjni, "A Depolarizing Chipless RFID Tag for Robust Detection and Its FCC Compliant UWB Reading System," in IEEE Transactions on Microwave Theory and Techniques, vol. 61, no. 8, pp. 2982-2994, Aug. 2013, doi: 10.1109/TMTT.2013.2267748.

10. V. P. Plessky and L. M. Reindl, "Review on SAW RFID tags," in IEEE Transactions on Ultrasonics, Ferroelectrics, and Frequency Control, vol. 57, no. 3, pp. 654-668, March 2010, doi: 10.1109/TUFFC.2010.1462.
11. Betancourt, D. et al. "Bending and Folding Effect Study of Flexible Fully Printed and Late-Stage Codified Octagonal Chipless RFID Tags." IEEE Transactions on Antennas and Propagation 64 (2016): 2815-2823.

12. S. Preradovic, I. Balbin, N. C. Karmakar and G. Swiegers, "A Novel Chipless RFID System Based on Planar Multiresonators for Barcode Replacement," 2008 IEEE International Conference on RFID, 2008, pp. 289-296, doi: 10.1109/RFID.2008.4519383.

13. Xu, Lei and Huang, Kama. (2015). Design of Compact Trapezoidal Bow-Tie Chipless RFID Tag. International Journal of Antennas and Propagation. 2015. 1-7. 10.1155/2015/502938.

14. Karmakar N.C., Kalansuriya P., Azim R.E., Koswatta R. Chipless Radio Frequency Identification Reader Signal Processing. John Wiley \& Sons, Inc.; Hoboken, NJ, USA: 2016.

15. Hunt V.D., Puglia A., Puglia M. RFID: A Guide to Radio Frequency Identification. Wiley-Interscience; Hoboken, NJ, USA: 2007.

16. Karmakar N.C., Amin E.M., Saha J.K. Chipless RFID Reader Architecture. John Wiley \& Sons, Inc.; Hoboken, NJ, USA: 2013. 